\title{
Growth Acceleration of Bean Sprouts by the Application of Electrochemical Voltage in a Culturing Bath
}

\author{
Yoshiyuki MIZUGUCHI, Yumi WATANABE, Hiroaki MATSUZAKI, \\ Yasunari IKEZAWA and Tsutomu TAKAMURA* \\ Received May 18, 1994 ; Accepted July 30, 1994
}

\section{INTRODUCTION}

Acceleration of plant growth by physical stimuli without chemicals will provide an attractive means not only of growing plants, but also of protecting the natural environment since chemicals used to stimulate growth frequently give rise to toxic and polluting problems in nature. It is well known that the living activity of plants is strongly related to the electrochemical potential difference across the cell membrane of a living cell ${ }^{1-3}$ ). The influence of electrochemical stimuli on cell activity has also been investigated in animals ${ }^{4}$ ). For the purpose of examining electrochemical effects on the growth of plants, we studied the application of electrochemical voltages to growing bean seedlings through an electrochemical culturing solution. When we applied dc voltage to the culturing solution, growth was found to be accelerated by about $30 \%$ in the case where about 1 volt was applied 5,6$)$. The accelerated growth rate was dependent on the applied voltage, and voltages higher than 4 volts retarded the growth. Growth acceleration was observed under the illumination of light, however, no acceleration was observed at any voltage in the dark.

Such an accelerating effect is considered to be related to the stimulation of the ion pump action for growth metabolism. It is worthwhile to examine what type of the stimulation (continuous or intermittent etc.) is most effective for the acceleration. We therefore investigated the effect of alternating cur-

Department of Chemistry, College of Science, Rikkyo (St. Paul's) University (Nishi-Ikebukuro, Toshima-ku, Tokyo 171 Japan)

Key Words: Growth Acceleration, Electrochemical Stimuli rent voltage application. In the present communication, we report on the results of the two cases of sinusoidal wave and square wave application.

\section{EXPERIMENTAL}

Bean seeds, Vigna Mungo (L) Hepper, were sterilized and cultured for 24 hours in an agar gel medium. Seedlings having 1-1.5 cm roots were planted in a floating agar plate and cultured in 0.5 mM potassium sulfate aqueous solution in acrylic polymer containers $(20 \times 7 \times 12 \mathrm{~cm})$ under controlled weather conditions. The container was separated into two equal compartments along the longer direction. In one of the compartments, two platinum plate electrodes were mounted at both sides separated by $15 \mathrm{~cm}$ and electrochemical voltage was applied from a voltage source during growth5). The actual values of voltage from place to place in the culturing solution were measured by using two $\mathrm{Ag} / \mathrm{AgCl}$ electrodes equipped with salt bridges, one of which was positioned near one of the Pt electrode. Nearly linear voltage gradient was found to exist between the two Pt electrodes in the culturing vessel showing no abrupt change near the Pt electrode compartment. Effects of chemical entities formed by the electrolysis were prevented by using agar plates to separate the electrode and cultivation parts. The other compartment was used as a control with no electrode. After culturing for 5 days under the illumination of light, we measured the plant body length. The growth ratio is defined by

Growth ratio $(\%)=\frac{\delta l_{\text {app }}-\delta l_{\text {con }}}{\delta l_{\text {con }}} \times 100$, and $\delta l=\frac{1}{\mathrm{n}} \sum_{i=1}^{\mathrm{n}}\left(l_{1}-l_{0}\right)_{i}$ 
where $l_{a p p}$ and $l_{\text {con }}$, are the lengths of the bean sprouts with and without applied voltage, respectively; $l_{0}$, the initial length; $l_{1}$, the final length of the bean sprout; $n$, the sample number.

\section{RESULTS and DISCUSSION}

With a sinusoidal wave of applied voltage the acceleration was found dependent not only on the frequency but on the amplitude. But only low level acceleration of at most $10 \%$ was observed. In contrast, when we applied a square wave, growth acceleration was quite remarkable. The results are depicted in Fig. 1, where the growth rate is plotted against the frequency with a 1 volt amplitude. As seen in the figure, a frequency of $20 \mathrm{~Hz}$ was the most effective. The effect of the amplitude was also examined for the case of $20 \mathrm{~Hz}$, and the results are shown in Fig. 2. With about 1 volt, the effect was most distinct. In this case, the acceleration rate was $30 \%$.

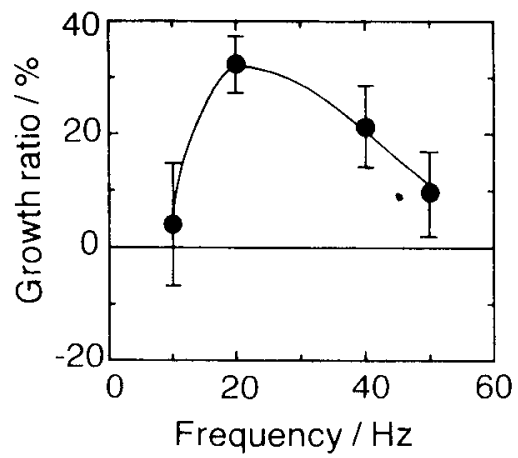

Fig. 1 Frequency dependence of bean sprout growth with the application of a square wave. Data represent means \pm S.E. of results from 60 sprouts sampled every frequency in four experiments.

The effect of a square wave was not restricted to primary root growth, but also promoted the shooting of lateral roots. This feature is shown in Fig. 3, which was photographed from the top. The lateral roots are seen as white lines centering at the primary root. Shooting of lateral roots enlarges the effective surface area of the root, which can take up more nutritious materials from the surrounding solution, resulting in the acceleration of the growth of the whole body of the sprout.

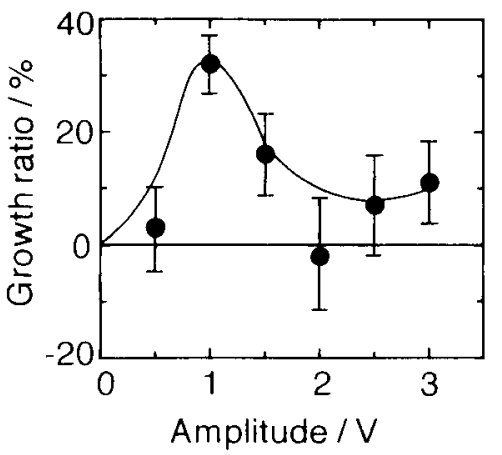

Fig. 2 Dependence of the growth ratio of bean sprouts on the applied square wave amplitude at $20 \mathrm{~Hz}$. Data represent means \pm S.E. of results from 60 sprouts sampled every amplitude in four experiments.

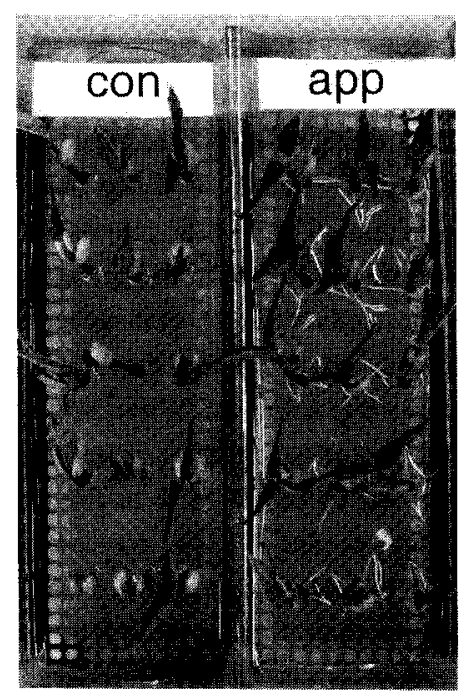

Fig. 3 Accelerated shooting of lateral roots by square wave application ( 3 volt, $20 \mathrm{~Hz}$ ).

The effect of voltage for the acceleration was observed only under the illumination of light. When we applied voltage, the acidity of the culturing solution was higher than that of control. It was confirmed that no acidity change occurred for the solution during the voltage application in the absence of sample specimen. Higher $\mathrm{H}^{+}$concentration found at the root of accelerated growth specimen is in accordance with the root growth mechanism explained by Toko et al. ). We suppose, therefore, as pointed out in the preceding communication, the fact that the 
acceleration was observed only under the illumination of light implies that the applied voltage assists photosynthesis of ATP. The reason why a square wave is the most effective is not clear at present, but is in accordance with the study by Kagawa and his coworkers ${ }^{4)}$ that the sudden change in the external electric field is effective to the $\mathrm{H}^{+}-$ATPase activation in rat liver mitochondria. In our case, the sudden change in the electric field in the solution is presumably able to stimulate $\mathrm{H}^{+}-$ATPase in mitochondria of the root cell, resulting in an activation of the ATP synthesis which supplies energy for the growth. As a result of the energy metabolism, $\mathrm{H}^{+}$is ejected from the system. In the case of sinusoidal wave, all the change is too slow to be effective in average so that the $\mathrm{H}^{+}-\mathrm{ATPase}$ activation is insufficient, resulting in the lower level acceleration.

The authors thank The Mitsubishi Foundation for their financial support of this work.

\section{REFERENCES}

1) C. J. Chastain and J. B. Hanson, Plant Science Letters, 24, 97 (1982).

2) A. H. De Boer, K. Katou, A. Mizuno and H. Okamoto, Plant, Cell and Environment, 8, 579 (1985).

3) Y. Nishizaki, Plant and Cell Physiology, 33, 1073 (1992).

4) T. Hamamoto, K. Ohno and Y. Kagawa, Journal of Biochemistry 91, 1759 (1982).

5) Y. Mizuguchi, Y. Ikezawa and T. Takamura, Denki Kagaku, 61, 255 (1993).

6) T. Takamura, Y. Mizuguchi, Y. Ikezawa and S. Takeuchi, Presented at the XVth International Botanical Congress Abstracts, 6056 (1993).

7) M. Souda, K. Toko, K. Hayashi, T. Fujiyoshi, S. Ezaki and K. Yamafuji, Plant Physiol., 93, 532 (1990). 